\title{
The anaphoric semantics of partial control *
}

\author{
Dag Trygve Truslew Haug \\ Department of Philosophy, Classics, \\ History of Arts and Ideas, University of \\ Oslo
}

\begin{abstract}
This paper deals with partial control, the phenomenon that instead of identity there is a subset relation between the controller and the controllee in a control construction. We argue that the context dependency of partial control requires an anaphoric approach, rather than hard-coding the possibility of a reference shift in the semantics of the control verb. The anaphoric analysis is formalized in Partial Compositional Discourse Representation Theory.
\end{abstract}

Keywords: control, anaphora, DRT, bridging

\section{The semantics of syntactic dependencies}

Formal theories of syntax in general offer two different mechanisms for establishing dependencies between two syntactic positions. This difference can prototypically be illustrated as in (1), which shows (simplified) Government and Binding-style analyses of control (a) and raising (b).
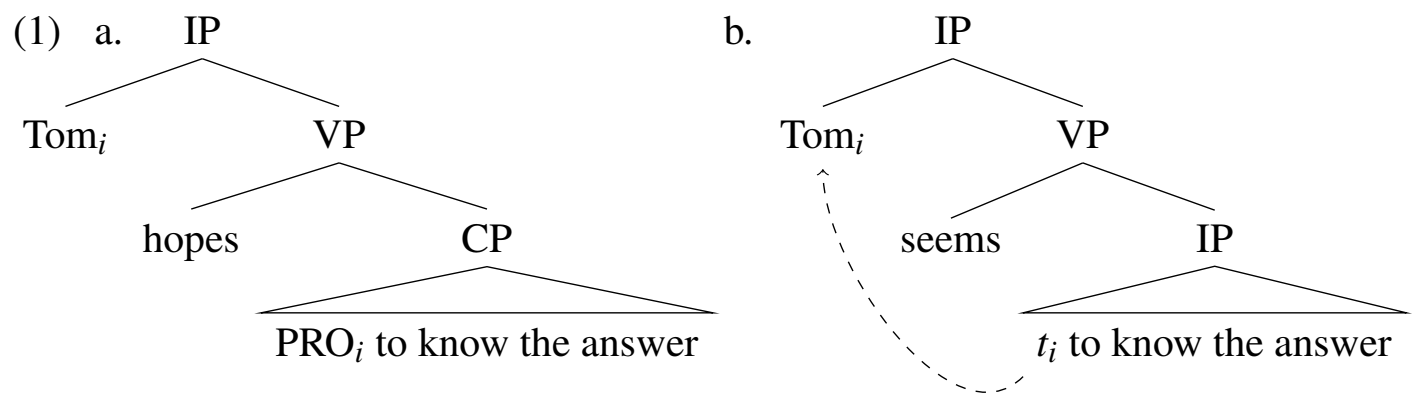

On this traditional analysis, control involves two distinct syntactic elements which are coindexed, forcing identity of their referential properties. In raising, by contrast, we have a single syntactic element, which fills multiple roles in the syntax. In the

* I thank the SALT audience and the members of the Oxford Glue Semantics seminar for valuable feedback.

(C2014 Haug 
derivational analysis sketched in (1b), this is implemented as movement, allowing the single element to occupy several syntactic positions during the derivation. In non-derivational frameworks such as LFG or HPSG, the same effect is achieved via structure sharing. For the purposes of this article there are no important differences between the two approaches, which we will refer to collectively as the identity analysis, as opposed to the coindexation analysis traditionally found in the analysis of control.

Coindexation always involve two semantically interpreted positions. Cases of syntactic identity, on the other hand, sometimes involve only one semantically interpreted position - as in raising, for example. But sometimes both positions are interpreted. Of particular interest here are identity analyses of control, i.e. approaches which assimilate control to raising (Bresnan 1982; Andrews 1982; Hornstein 1999; Boeckx, Hornstein \& Nunes 2010).

Standard analyses (e.g., Heim \& Kratzer 1998) make no difference between syntactic positions that are linked via coindexation and those linked through identity. Concretely, these dependencies are uniformly treated via variable binding. (2) shows some sketch analyses involving variable binding.

$$
\begin{array}{lll}
\text { relativization } & \lambda x \cdot P(x) \wedge Q(x) & (P=\text { head noun, } Q=\text { relative clause }) \\
\text { binding } & \exists / \forall / \lambda x . \Phi(x, x) & (\Phi=\text { some (complex }) \text { formula }) \\
\text { control } & \lambda x . P(x, Q(x)) & (P=\text { control verb, } Q=\text { control infinitive })
\end{array}
$$

Such analyses are possible because elements like PRO and (bound) pronouns are interpreted as variables rather than content items; and traces of movement likewise.

The semantics in (2) predicts that the top and the bottom of the dependency are fully covariant, since they are represented by the same variable. And yet we do find cases which prima facie run counter to this prediction.

$$
\begin{aligned}
& \text { adjjmag } \chi^{\mathrm{w}} \partial z ̌ d 3 r \text { sə } \text { vžag-əl }_{i} \quad \text { zur-a, } \\
& \text { person better what language-SUPER speak-SBJV.3SG }
\end{aligned}
$$

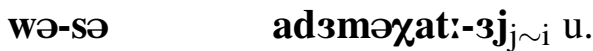

DemDist-ATTR nationality-ABL be.PRS.3SG

'A person belongs to the nation whose language s/he speaks better.' (lit. 'What language $\mathrm{a}_{\mathrm{i}}$ person speaks better, from that nation $_{\mathbf{j} \sim \mathrm{i}}$ he is.')

(4) In every ${ }_{i}$ room the $j \sim i$ patient has someone visiting.

(5) $\quad$ The $i$ chair wants $\mathrm{PRO}_{j \sim i}$ to gather at six.

(3) illustrates the structure of an Ossetic correlative clause (Belyaev \& Haug 2014). What is crucial for us here is that there cannot be an individual which at the same time satisfies the properties denoted by both the relative clause and the head noun, as the analysis in (2) would predict. Similarly, the quantifier every room in (4) binds 
Partial control

the patient; the two positions co-vary, but there is no identity relation between them, as (2) predicts. Finally, (5) illustrates partial control, where the controller and the controllee position in a control chain are not identical: the matrix subject the chair is singular, but the controlled subject position must be plural due to the collective predicate gather.

One way around this problem is to assume implicit material, as illustrated in (6)-(8).

(6) Which ${ }_{i}$ language a person speaks better, from the nation (with that ${ }_{i}$ language) he is.

(7) In every ${ }_{i}$ room the patient (in that ${ }_{i}$ room) has someone visiting.

(8) The $\mathrm{T}_{i}$ chair wants $\mathrm{PRO}_{i}$ to gather (with the committee) at six.

However, another strategy for dealing with these cases is to view them as instances of bridging (Clark 1975; Asher \& Lascarides 1998), i.e. anaphoric dependencies that involve partial coreference between related concepts such as language nation, room - patient (in a hospital setting), chair - committee. This is the strategy that I will pursue in this paper. I will not spend much time on the 'silent comitative' analysis illustrated in (8) as it has been extensively discussed in the syntactic literature and is rejected by most researchers. However, there is another analysis which involves a silent 'shifting' of the interpretation of the subject position. The basic idea, illustrated in (9), is found in Asudeh 2005.

want: $\quad \lambda x . \lambda P . \exists y \cdot w a n t(x, P(y) \wedge x \subseteq y)$

An analysis along this line has recently been proposed by Pearson (to appear), which is the first and only in-depth semantic study of partial control and therefore the obvious starting point for further analysis. Nevertheless I will argue that the shifting analysis is not tenable and that an anaphoric approach should be taken instead.

The structure of the paper is as follows. In section 2, I look closer at the phenomenon of partial control. Section 3 discusses the shifting hypothesis and why it is problematic. The problems lead to an anaphoric approach, developed in section 4 , which is able to capture the context-dependency of partial control. Finally, section 5 offers conclusions as well an outlook on the nature of partial control predicates.

\section{The phenomenology of partial control}

The basic observation, stressed by Landau $(2000,2004)$ is that some but not all control predicates allow the embedding of a collective predicate even when the subject is singular:

(10) a. ? The chair wanted to gather at six. 
b. ? The chair preferred to gather at six.

c. ? The chair agreed to gather at six.

(11) a. * The chair tried to gather at six.

b. * The chair began to gather at six.

c. * The chair managed to gather at six.

As has often been observed by Landau and many others, partial control is somewhat marginal and requires contextual support - here in the form of the subject the chair which primes a semantic plurality, a committee or a similar body that the chair is chairing. However, even to speakers who do not like (10) the contrast in acceptability between (10) and (11) is robust and points to a split between predicates that allow partial control (henceforth PC predicates) and those that do not (henceforth EC (exhaustive control) predicates).

Moreover, Landau (2000) observes that the plurality in the embedded subject position is semantic only, not syntactic. This is shown by the fact that partial control does not license a plural anaphor, as illustrated in (12).
a. * The chair wanted to meet each other.
b. * The chair preferred to meet each other.
c. * The chair agreed to meet each other.

Another fact that has been made much of in the literature (again, the observation goes back to the pioneering work of Landau 2000) is that partial control seems to correlate with the ability to shift the time in the complement clause.

(13) a. Yesterday, the chair wanted to hold the meeting tomorrow.

b. Yesterday, the chair preferred to hold the meeting tomorrow.

c. Yesterday, the chair agreed to hold the meeting tomorrow.

a. * Yesterday, the chair tried to hold the meeting tomorrow.

b. * Yesterday, the chair began to hold the meeting tomorrow.

c. * Yesterday, the chair managed to hold the meeting tomorrow.

Finally, it has been shown that partial control forces a de se-reading, i.e. not only is the controller partially coreferent with the controllee, but the controller must also be aware of this fact. In other words, partial control (like exhaustive control) is not compatible with a 'mistaken identity' scenario as in (15), from Pearson 2013: 307.

(15) John is an amnesiac. He is watching footage of an Olympic figure skating competition in which he competed, although he has forgotten this fact, and 
Partial control

does not even recognise himself on the screen. He says 'I think that team is going to win the medal, look how well they work together.'

We can report this felicitously with (b) but not (a).

a. \# John expects to win the medal by working well together.

b. $\mathrm{John}_{i}$ expects that they ${ }_{i+}$ will win the medal by working well together.

This is important because de se is characteristic for obligatory control, whereas non-obligatory control structures do not show this effect (Hornstein 2003: 13-14). Partial control must therefore be a case of obligatory control and we cannot get around the partial coference by assuming that the referential relationship between the controller and the controllee is free.

\subsection{The acceptability of partial control}

The last section presented core grammatical data about partial control, which any theory must derive. Another fact of a more pragmatic nature is that partial control requires contextual support and that even in the best of contexts, partial control examples are somewhat marginal and degraded. The clearest effects of this contextdependency are in fact shown by a number of false predictions that the shifting analysis makes, so we will return to this in section 3 . For now we focus on the acceptability of partial control.

The most important study on this subject is the experiment reported in White \& Grano 2013, where participants were asked to rate the acceptability of partial control on a scale from 1 to 7 . The form of the test sentences along with some examples are given in 16.

(16) X said that Y P-ed to Q/Q-ing (together).

a. Jan said that Jerry wanted Beth to rent an apartment in Baltimore (together).

b. Betty said that Val hoped to take yoga classes (together).

c. Erin said that Sal remembered renting an apartment in Baltimore (together).

The key question, then, is how the acceptability of sentences varies as a function of the presence or absence of together with different predicates in the $\mathrm{P}$ position. White \& Grano (2013) report the results in Figure 1. The baseline reported here are sentences like "X said that Y Q-ed (together)" (e.g., "Betty said that Val took yoga classes (together)"). As Figure 1 suggests, there is a group of control predicates (on the left side of the figure) which do not seem to tolerate PC in any form. It should be stressed that Figure 1 shows the raw mean ratings, which need to be corrected for various error sources in order to be compared to the baseline. However, 


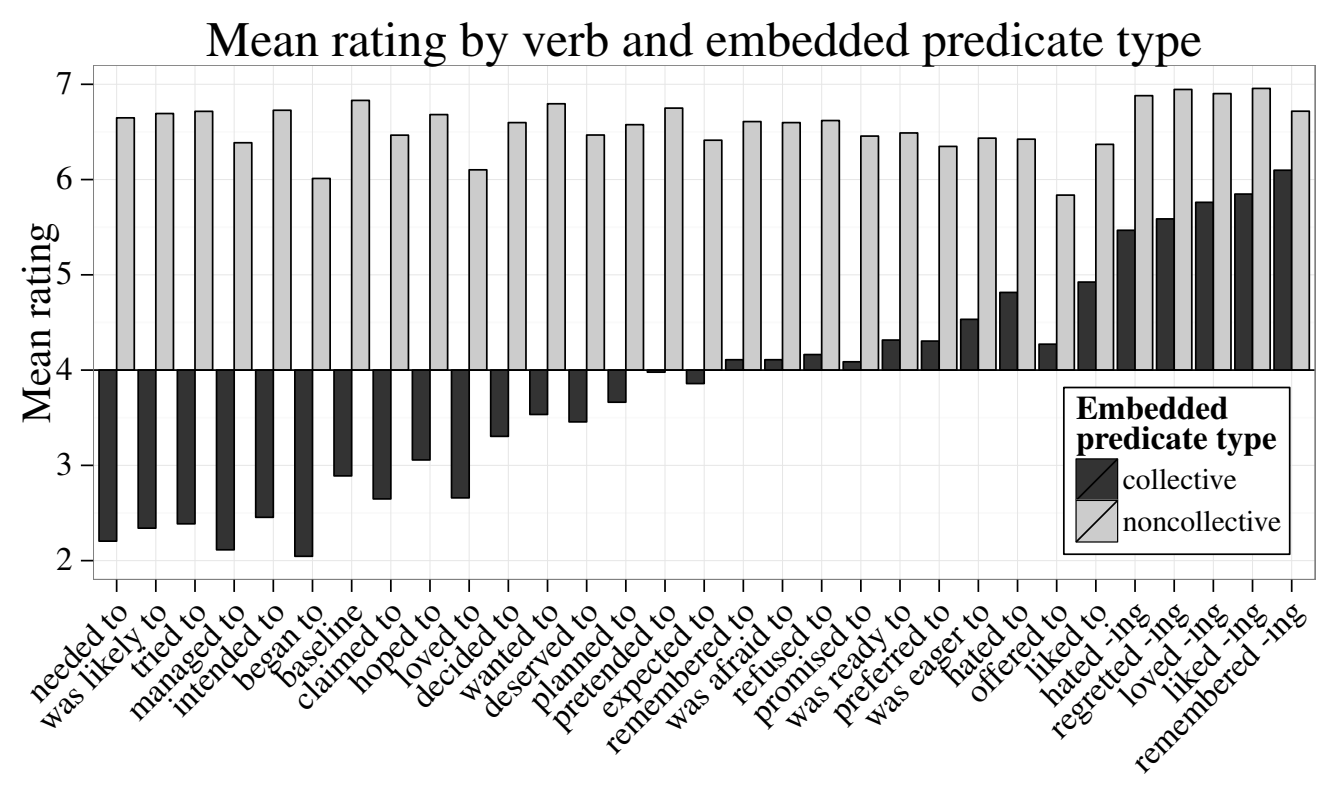

Figure 1 Acceptability of PC according to White \& Grano (2013)

what Figure 1 does show quite clearly is that all predicates - including those on the righthand side - are to some extent degraded in the PC construction: the difference in rating between collective and noncollective examples is statistically significant for all predicates (Wilcoxon test, $p<0.005$ ).

This confirms that PC is somewhat marginal even with the predicates that tolerate it, suggesting that we should not aim for a semantics which directly licences PC, as the shifting approach in (9) does. Instead, PC looks like a pragmatic reinterpretation, a repair strategy which is available with some control predicates in cases where a singular interpretation of the embedded subject position does not make sense. This also accounts for the context dependency of PC. It is worth noting that although White \& Grano (2013) did not test the effects of context on the acceptability of PC, they did make sure that all their examples provided a salient plurality. In the next section, we will see how the context sensitivity of PC plays out in various ways that contradict the predictions of the shifting approach.

\section{Why shifting fails}

We have already seen the most basic problem of the shifting approach: it predicts that PC examples are straightforwardly grammatical and not degraded in any way, 
contrary to intuitions and to the experimental data in White \& Grano 2013. Another way to look at this is to consider the parallel with time shift. We saw above that there is a connection between the availability of PC and the possibility of shifting the time in the complement clause. Nevertheless, it is intuitively clear that the backward time shift in the complement of remember $X$-ing and the forward time shift in the complement of expect is part of the lexical semantics of these verbs in a way that the shifting of the subject is not, against the predictions of the shifting analysis.

This conceptual problem translates into a number of wrong predictions which we will now look at in more detail. Before we do that, we need to present the details of the shifting analysis, as elaborated by Pearson (to appear).

\subsection{Centered worlds and extensions}

Pearson (to appear) analyses control verbs in terms of centered worlds, which are basically an enrichment (first proposed by Lewis 1979) of the ordinary possible worlds approach to intensionality. On this approach the intensional parameter is not just a world, but something more like a Kaplanian context, a triple $\left\langle c_{W}, c_{A}, c_{T}\right\rangle$ consisting of a world $c_{W}$, an agent $c_{A}$ in that world, and a perspective time $c_{T}$. Modal alternatives are then defined in terms of this construction, e.g., bouletic alternatives are as in (17), and the semantics of a PC verb such as want is defined as in (18).

want $_{x, t, w}:=$ the set of centered worlds $c$ such that it is compatible with $x$ 's desires at $t$ in $w$ for $x$ to be $c_{A}$ in $c_{W}$ at $c_{T}$

$\llbracket \mathrm{x}$ wants $\mathrm{P} \rrbracket=1$ iff for all of $x$ 's want-worlds $c$ there is an extension $c^{\prime}$ such that $c_{A}^{\prime}$ has the property $P$ at $c_{T}^{\prime}$ in $c_{W}^{\prime}$

(18) makes crucial appeal to a notion of extension, which is defined in (19).

$$
\begin{aligned}
c^{\prime} \text { extends } c \text { iff } \\
\text { - } c_{W}=c_{W}^{\prime} \\
\text { - } c_{A} \subseteq c_{A}^{\prime} \\
\text { - } c_{T} \subseteq c_{T}^{\prime} \text { or } c_{T}<_{\text {precedes }} c_{T}^{\prime} \text { or } c_{T}>_{\text {precedes }} c_{T}^{\prime}
\end{aligned}
$$

This approach 'bundles' the extension of the controlee denotation and the shifting of the time in the control complement. But it is not clear that this bundling yields an insightful unification of the phenomena, for it is essentially a conjunction of different conditions. ${ }^{1}$

1 Pearson (to appear) formulates the concept in a slightly different way, by assuming that the definition given above for the extension of the time coordinate in principle applies to the world and agent coordinates too, but reduces to the relations given above because precedence does not apply to worlds 
And in fact, it is not clear that we should attempt to bundle the shifting of the subject and time arguments, because the evidence suggests that these operations are quite distinct. The time shift in the complement clause is obligatory: No amount of contextual support can make us 'remember' future events or 'expect' past ones. ${ }^{2}$ This means that it is appropriate for the semantics of these verbs to introduce a distinct, existentially quantified time argument restricted to the past and the future respectively (and possibly further constrainted by temporal adjuncts etc.). On the other hand, the shifting of the subject position is heavily constrained by contextual factors. This means that a semantics which builds the shifting into the semantics via existential quantification (either directly over the subject argument or indirectly over extensions of centered worlds) is too weak, since it both fails to account for the context-dependency and predicts that partial control is not in any way degraded, contrary to the results of White \& Grano (2013).

\subsection{Scope problems}

In addition to the general problem of overgenerating described above, the hardcoding of the shifting in the lexical semantics leads to rigid scope predictions. In particular, since extensions are part of the verb meaning, it is predicted that they always take low scope relative to operators that outscope the verb. Moreover, the lexical semantics of the verb must fix the relative scope of the extension part and the modal part of the meaning. We will now look closer at these problems.

\subsubsection{Quantification}

Consider (20).

(20) Everybody wanted to have lunch together.

The extension approach yields the meaning in (21).

(21) $\forall x . \forall c \in$ want $_{x, n, w}$ there is an extension $c^{\prime}$ of $c$ such that the center of $c^{\prime}$ has lunch together in $c^{\prime}$

In (21), the universal quantifier $\forall x$ outscopes the verbal meaning and hence the quantification over extensions. This means that we can paraphrase (21) as "For all $x$, $x$ wants that there is a plurality $y \supseteq x$ such that $y$ has lunch together", i.e. we predict a distributive reading.

and individuals and because worlds do not form pluralities, so that $c_{W} \subseteq c_{W}^{\prime}$ entails $c_{W}=c_{W}^{\prime}$. Even so, the definition of extension is a disjunction of relations, some of which do not apply to all coordinates, showing that there is no real unified concept at work.

2 Though there is a distinct meaning of expect as a synonym of suppose. 
Partial control

But (20) does not have a distributive reading of the subject extension in the absence of contextual support (as in e.g., 'Every chair wanted to gather at six.') However, the subject quantifier can easily distribute over the time of the complement clause. (This reading can be brought about by supplying e.g., 'But they all had different time preferences.) This means that we should be aiming at a semantic representation as in (22) rather than (21).

$$
\forall x . \forall c \in \text { want }_{x, n, w}, \exists t>n \text { such that } x^{\prime} \text { has lunch in } w \text { at } t
$$

where $x^{\prime}$ is some contextually salient plurality, in this case the restrictor set of the quantifier, which is the same for all $x$.

\subsubsection{Modality and modal subordination}

Consider (23) and the asssociated Pearson-style semantics in (24)

(23) John is lonely. He wants to have lunch together.

(24) $\forall c \in$ want $_{j, n, w}$ there is an extension $c^{\prime}$ of $c$ such that the center of $c^{\prime}$ has lunch together in $c^{\prime}$

As (24) shows, the existential quantification over extensions is in the scope of the modal component of the verb (the universal quantifier over bouletic alternatives). We can paraphrase (24) with "John wants that there is some plurality $y \supseteq$ john such that $y$ has lunch together" or, somewhat sloppily, "John wants someone to have lunch with".

But (23) cannot mean that. It can only mean that John wants that some contextually salient plurality (including himself) has lunch together. In (23) we can construct such a plurality by including discourse participants, yielding the meaning "John wants to have lunch with me/you/you and me."

However, while the controlled subject position in (23) must clearly outscope modality, this is not always the case. Partial control can also occur in contexts of modal subordination, where the salient plurality exists only in the scope of the modal context, as in (25).

(25) John is looking for a group of elves. He wants to have lunch together.

The context dependency that we saw in (20) and (23) suggests that partial control involves anaphora. Modal subordination is standardly taken to involve anaphoric retrieval of a discourse referent introduced within the scope of a prior modal expression, so (25) also supports the theory that partial control involves anaphora. However, we cannot simply say that the controlled subject position resolves to $a$ group of elves or even to a split antecedent consisting of he and a group of elves, since 
obligatory control (of which partial control is a subtype) does not allow sentenceexternal antecedents. A more sophisticated anaphoric approach is needed, relying on a notion of bridging. In the next section, we develop this idea and show how it can be formalized in a variant of Compositional Discourse Representation Theory.

\section{An anaphoric approach}

The idea behind the anaphoric approach to partial control is to assimilate the indirect referential relationship that we find between PRO and its controller to the indirect referential relationships that we find in discourse anaphora.

(26) $\mathrm{The}_{i}$ chair wants $\mathrm{PRO}_{j \sim i}$ to gather at six.

(27) The ${ }_{i}$ chair is happy. The ${ }_{j \sim i}$ committee met at six.

This means that we view PRO as a contentful item, an anaphoric expression that contracts a (possibly indirect) referential relationship to its antecedent, although unlike in the case of discourse anaphora - that antecedent is assigned by the grammar via the mechanism of control, rather than resulting from pragmatic inference.

Moreover, PRO is a pronoun, not a DP. From a DRT perspective this is not an essential semantic difference; pronouns are basically semantically attenuate definite descriptions (Geurts 2011). And the lack of descriptive material constrains bridging with pronouns. As observed by Nouwen (2003: 73), example (28) is infelicitous, in spite of an impressive amount of contextual support.

(28) We cannot use John's car. \#All four of them are flat.

However, it is not the case that bridging is always infelicitous with pronouns, as the following examples show.

(29) My next-door neighbours make a lot of noise. He plays the drums and she keeps on shouting at him.

(30) John kept on staring at the newly-wed couple. She resembled a childhood sweetheart of his.

(31) The priest was tortured for days. They wanted him to reveal where the insurgents were hiding out.

(32) When Little Johnny threw up, was there any pencil-eraser in it?

Nouwen (2003: 74-76) identify the following conditions on pronominal bridging:

- inferability

- uniqueness 
Partial control

- use of semantically available information only

- support of discourse coherence by the anaphoric link

Inferability is a straightforward condition which applies to all instances of bridging. The coherence condition rules out pronominal bridging in less coherent discourse contexts such as in the following examples.

\#My next-door neighbours make a lot of noise and I met her yesterday.

(34) \#John kept on staring at the newly-wed couple. She was thinking about the flowers.

(35) \#The priest was tortured for days. They went for beers every day.

This constraint seems to restrict pronominal bridging more than bridging with full DPs. But in the case of control, the grammar specifies the relationship between the antecedent clause (containing the controller) and the control complement, so that we can assume that the constraint is not relevant.

The second and the third constraints are therefore the most relevant for our purposes. Together they amount to a requirement that the referent of the pronoun must be semantically unique. For example, the set of wheels is a pragmatically salient, unique set of cardinality four associated with most cars, but this is not enough to licence bridging in (28). The claim, then, is that bridging fails here because the relation 'car' - 'set of four wheels' is not encoded in the semantics of car, but relies on pragmatic reasoning.

There is more research to do before Nouwen's observations can be developed into a full theory of pronominal bridging (it is for example unclear how neighbours in (29) introduce a unique male and a unique female), and yet more before we can claim to know how a bridging theory of partial control can constrain the acceptability of this phenomenon in various contexts, with different controllers and different control predicates. But the anaphoric theory is at least consistent with some basic facts about partial control, such as the variability in judgements and the fact that it looks like a pragmatic 'repair' strategy that is only licensed when the literal exhaustive control reading does not make sense; we prefer fully coreferent resolutions of pronouns.

It is crucial to note that partial control is like bridging with pronouns, not like bridging in general. With full DPs, the antecedent every doctor can license an anaphor the patient as in (36), but this is impossible with a pronoun (37) and with PRO (38).

(36) Every doctor wants the patient to get better.

(37) Every doctor wants him (= the patient) to get better.

(38) Every doctor wants PRO (= the patient) to get better. 
The pronominal bridging theory predicts this because the semantics of doctor does not introduce a unique patient. Some other observations also follow. For example, (Landau 2000: 7) observed that there is no "superset control", so that (39) is ungrammatical.

(39) $\mathrm{The}_{i}$ chair was glad the ${ }_{j}$ committee had agreed to $\mathrm{PRO}_{i \subset j}$ wear a tie.

The intended reading here is that the committee had agreed that the chair would wear a tie, which is ungrammatical. The ungrammaticality is expected on Nouwen's theory because a plausible representation of the lexical semantics of committee does not include a unique individual to which PRO could refer (although the committee chair may be pragmatically more salient). On the other hand, the semantics of chair likely does include reference to the unique plurality being chaired, thereby licensing the bridging in (5).

\subsection{The antecedent of PRO and feature (mis-)matching}

Since PRO is a semantically dependent item (whether a bound variable as per the traditional analysis, or an anaphoric pronoun as assumed here), it must have an antecedent. There are two possible candidates. The traditional view is that the controller is the antecedent of PRO. A more recent view (Schlenker 2003; Maier 2009; Stephenson 2010) is that PRO resolves to the center of the attitude in which it appears. It is often assumed that PRO denotes the attitude center directly, but partial control shows us that this cannot be the case. In the figure skating scenario in (15), John is the attitude center and has a de se belief about himself, but PRO refers to the entire team. One way out of this, taken by Stephenson (2010) is to assume a "plural PRO" which refers to a plurality containing the attitude center, much like we refers to a plurality including the speaker. However, this approach overgenerates in the sense that it predicts that partial control is straightforwardly grammatical. It also leaves unexplained why PRO in partial control is not syntactically plural.

In fact, as pointed out by Landau (2000); Schlenker (2003) and others, PRO always bears the agreement features of its controller. We therefore assume that PRO takes the controller as its antecedent. This antecedent is imposed by the grammar (although its reference can be affected by bridging), which means that PRO is a bound pronoun. And precisely because it is a bound pronoun, there can be a mismatch between the agreement features, which reflects those of the binder, and the semantic features. This is also attested with overt bound pronouns, as in (40) (from Uli Sauerland, cited in Schlenker 2003: 96n61) where the semantics of the embedded predicate makes clear that the second we must be singular despite the plural morphology.

(40) We all sometimes think we are the only person in the world. 
Partial control

\subsection{Formalization}

\subsubsection{Partial Compositional Discourse Representation Theory}

Partial Compositional DRT (PCDRT, Haug 2013) is a reformulation of Muskens' Compositional DRT (CDRT, Muskens 1996) within a partial logic. The goal is to be able to deal with anaphora without using coindexation, while maintaining the desirable aspects of CDRT, in particular compositionality through lambda calculus and the ability to abbreviate complex expressions of the 'logic of change' as regular DRSs, familiar from the original DRT language (Kamp 1981; Kamp \& Reyle 1993; Kamp, van Genabith \& Reyle 2011).

The crucial innovation in CDRT is to inject discourse referents (and assignments) into the object language as entities of their own type, rather than to treat them as simply variables over ordinary type $e$ individuals. This means that we talk about discourse referents and their antecedents in the logic itself and not only in the metalanguage. This possibility is not exploited in Muskens' original work, where anaphoric effects are instead captured by using the same discourse referent constant for the anaphor and the antecedent, which will therefore be coreferent under any assignment. But this approach requires syntactic coindexation of the anaphor and the antecedent.

PCDRT instead underspecifies anaphoric relationships. In the general case, all that needs to be said is that an interpretation must give all anaphoric discourse referent accessible antecedents in order to make sense. The concrete anaphoric resolution is supplied by a function $\mathscr{A}$ which takes anaphors to antecedents. $\mathscr{A}$ is supplied by pragmatic inference, although it is constrained by semantic factors such as accessibility. For example, after processing (41) we may entertain the resolution that $x_{4}=x_{1}$ (on the assumption that John did something stupid because he was drunk), giving us the representation in (42). The overlining of the discourse referent $x_{4}$ indicates that it is anaphoric and must find an antecedent. The actual antecedent is provided by $\mathscr{A}$, which is not part of the semantic content.

(41) John 1 hid Bill's key $_{3}$. He 4 was drunk.

\begin{tabular}{|c|c|}
\hline $\begin{array}{llll}x_{1} & x_{2} & x_{3} & \bar{x}_{4}\end{array}$ & \\
\hline john $\left(x_{1}\right)$ & \\
\hline $\operatorname{bill}\left(x_{2}\right)$ & \\
\hline$k e y\left(x_{3}\right)$ &, $\mathscr{A}=\left\{x_{4} \mapsto x_{1}\right\}$ \\
\hline $\operatorname{poss}\left(x_{2}, x_{3}\right)$ & \\
\hline $\operatorname{hide}\left(x_{1}, x_{3}\right)$ & \\
\hline $\operatorname{drunk}\left(x_{4}\right)$ & \\
\hline
\end{tabular}


If we update (41) with (43), this attaches most coherently to the discourse if we take it to be an explanation, i.e. John hid Bill's key because Bill shouldn't drive in his present state of inebriation. This means we non-monotonically change the resolution of $x_{4}$ to $x_{2}$ instead of $x_{1}$, yielding the representation in (44)

(43) So he 5 shouldn't drive.

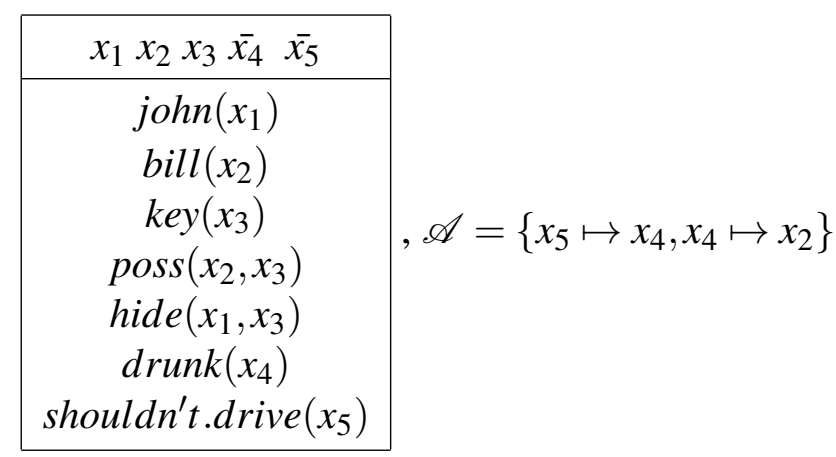

The point is that the DRS only tracks monotonic content, while non-monotonic content - including anaphoric resolutions - is modelled as a set of inferences that be recomputed and destructively updated. The presentation here is simplified since it assumes that at any stage, the interpreter only considers one possible antecedent for each anaphoric expression. In a more realistic setting, $\mathscr{A}$ would be a probability distribution over possible antecedents, but this distribution would nevertheless have to be updated non-monotonically.

Second, the version presented above assumes that anaphors and antecedents are always fully coreferent. For our purposes, we need to capture bridging relationships as well, and we do this by enriching the pragmatic component with a function $\mathscr{B}$ taking anaphors to a relation between individuals. The anaphor-antecedent relationship between $x_{\text {anaph }}$ and $x_{\text {ant }}$ is satisfied in the model iff $\mathscr{B}\left(x_{\text {anaph }}\right)$ holds of $\left\langle x_{\text {anaph }}, x_{\text {ant }}\right\rangle$. As an example, consider (45), with the analysis in (46). Notice the use of $\partial$, Beaver's unary presupposition operator (Beaver 1992).

(45) John entered the room. The chandelier sparkled brightly.

$$
\begin{array}{|c|}
\hline x_{1} \overline{x_{2}} \overline{x_{3}} \\
\hline \begin{array}{c}
\text { john }\left(x_{1}\right) \\
\partial\left(\operatorname{room}\left(x_{2}\right)\right) \\
\text { enter }\left(x_{1}, x_{2}\right) \\
\text { spark.brightly }\left(x_{3}\right) \\
\partial\left(\text { chandelier }\left(x_{3}\right)\right)
\end{array}
\end{array}, \mathscr{A}=\left\{x_{3} \mapsto x_{2}\right\}, \mathscr{B}=\left\{x_{3} \mapsto \lambda x \cdot \lambda y \cdot \operatorname{in}(x, y)\right\}
$$


We see that in this case, the pragmatics provides not just a resolution of $x_{3}$ to $x_{2}$, but also a referential relationship between the two referents. A uniqueness presupposition could also be added, but we omit a detailed analysis of the semantics of definiteness here. The DRS $K$ in (46) will be interpreted as a relation between input and output assignments and given PCDRT's truth definition, it will be true iff for the empty input assignment $i$, there exists an output assignment $o$ such that $K(i)(o)$ is true (which means that $o$ extends $i$ with assignments for $x_{1}, x_{2}, x_{3}$ and all conditions of $K$ are verified in $o$ ) and $o$ verifies $\operatorname{in}\left(x_{3}, x_{2}\right)$. For more details, I refer to Haug (2013).

\subsubsection{A PCDRT analysis of partial control}

To deal properly with control verbs, it is necessary to move to an intensional semantics. For this, we follow Maier (2009) and assume that DRSs denote sets of centered worlds $\langle a, w\rangle$ (in CDRT terms, DRSs now abbreviate terms of type (sseit), where $s$ is the type of assignments/states, and $i$ is the type of worlds). This means that in any DRS, there is an interpretable singleton predicate center. With this in place, we can define the syntax and semantics of intensional verbs as in (47)-(48).

$$
\begin{aligned}
& \text { If } x \text { is a dref and } K \text { a DRS, } w a n t(x, K) \text { is a DRS condition } \\
& \llbracket \text { want }(x, K) \rrbracket \text { is true iff } \text { want }_{x} \subseteq \llbracket K \rrbracket
\end{aligned}
$$

where want $_{x}$ denotes the set of centered worlds $\langle a, w\rangle$ such that it is compatible with $x$ desires for $x$ to be $a$ in $w .^{3}$

We are now ready to spell out the meanings of the lexical items involved in a typical partial control configuration such as (5).

3 Strictly speaking, want $t_{x}$ should be relativized to the current world, but we simplify here. 
PRO:

wants:

the chair:

to gather at six:

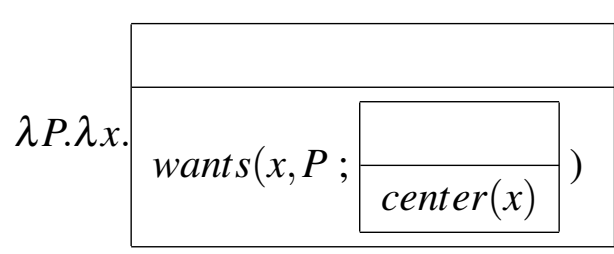

$\lambda P . \quad \overline{x_{1}}: P\left(x_{1}\right)$

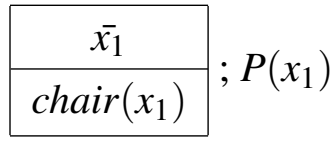

\begin{tabular}{l|c|}
$\lambda$. & $\overline{x_{2}}$ \\
\cline { 2 - 2 } & $\mathscr{B}\left(x_{2}\right)\left(x_{2}, \mathscr{A}\left(x_{2}\right)\right)$ \\
center $\left(\mathscr{A}\left(x_{2}\right)\right)$
\end{tabular}$; P$

$\lambda x$

A couple of points are worth mentioning about this semantics. Consider first PRO. The core of this meaning is a standard generalized quantifier: it takes a predicate $P$, introduces a discourse referent $x_{2}$, and applies $P$ to $x_{2}$. But it also introduces two conditions. First, $\mathscr{B}\left(x_{2}\right)\left(x_{2}, \mathscr{A}\left(x_{2}\right)\right)$ says that the bridging relation between $\mathrm{PRO}$ and its antecedent must hold; this has the effect of making the bridging relation hold $d e$ dicto, in the scope of the control verb. So, if The chair wants to gather at six is true, then in all of the the chair's want-worlds, the committee gathers at six and the chair chairs the committee, i.e. there are no want-worlds where the committee gathers at six but is chaired by someone else. This captures the aboutness component of de se attitudes (Pearson to appear): the attitude is genuinely about the attitude holder, not just about the referent of PRO, which on this analysis is not strictly coreferent with the attitude holder. Second, PRO introduces a condition center $\left(\mathscr{A}\left(x_{2}\right)\right)$, which makes sure that PRO resolves to the attitude center (though it is not necessarily fully coreferent with it). This reflects the awareness component of de se attitudes (again following Pearson (to appear)).

The semantics of want is relatively straightforward: it takes a subject $x$ and a proposition (DRS) $K$ and says that $x$ and $K$ stand in a want relation. But it also augments $K$ with the condition that $x$ must be the center of $K$. This is not specific to the control use of want: the subject of want will be the attitude center also of finite complements, although it will not necessarily bind a referent there.

Putting the semantics in (49) together in the obvious way, we get (50). 
Partial control

\begin{tabular}{|c|}
\hline \multicolumn{1}{|c|}{$\overline{x_{1}}$} \\
\hline want $\left(x_{1}, \quad \begin{array}{c|}\text { chair }\left(x_{1}\right) \\
\text { chairs }\left(\mathscr{A}\left(x_{2}\right), x_{2}\right) \\
\text { center }\left(\mathscr{A}\left(x_{2}\right)\right) \\
\text { centher.at } \operatorname{six}\left(x_{2}\right) \\
\text { gather. }\end{array}\right.$ \\
\hline
\end{tabular}

Since center is a singleton predicate (in each DRS), we can deduce from (50) that $\mathscr{A}\left(x_{2}\right)=x_{1}$. Put another way, the grammar - and in particular, the semantics specify the antecedent of PRO. In other words, we get a semantic theory of control. We cannot consider transitive control verbs in any depth here, but note that on the obvious generalization of this approach, we predict that the controller will be that argument of the control verb which is specified as the attitude center. So we can capture the object control behaviour of e.g., persuade if we assume that its meaning is something along the lines of $x$ (the subject) communicates with $y$ (the object) in such a way as to cause $y$ to intend $K$ (the complement).

If we simplify (50) using the equality $\mathscr{A}\left(x_{2}\right)=x_{1}$ which follows from the uniqueness of center, we get the representation in (51).

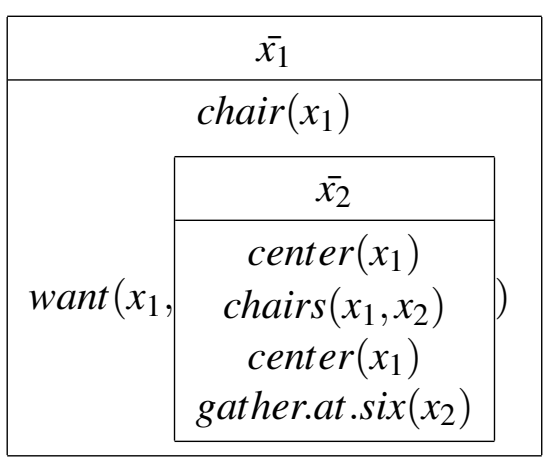

This is an adequate semantics for (5), and because of the flexibility of the anaphoric approach, it can easily capture the context-dependencies discussed in section 3.2.

\section{Conclusions and outlook}

We have argued for an anaphoric approach to partial control, which views it as a pragmatic repair phenomenon licensed by the grammar in cases where the fully coreferent interpretation of the controller and the controllee does not make sense. 
In many ways, our approach parallels Nouwen's account of complement anaphora: "This account, where complement anaphora are considered to be an extra-ordinary case of anaphora, might (at least partly) explain where this discomfort with pronominal reference to the complement set comes from" (Nouwen 2003: 79). Moreover, we get an account of why "superset control" is not possible, since this would seem to violate restrictions on pronominal bridging. Pronominal bridging remains ill understood and more research is needed on how it is constrained. Nevertheless, the anaphoric approach is compatible with the variable acceptability of partial control, and also flexible enough to account for the scope facts. It also answers some other questions that have been raised, such as why there is no partial raising: this follows directly from there being no pronoun involved in raising.

This points to a conceptual advance of the anaphoric approach more generally: it lets us define a semantic correlate to the distinction between syntactic coindexation and syntactic identity. This can be succesfully modelled in PCDRT because of the sophisticated account of anaphora and the distinction between monotonic content (in this case the binding of PRO) and non-monotonic content (in this case the possible bridging inference). By contrast, approaches (including the original CDRT of Muskens 1996) which simply reuse a variable to model anaphora are unable to draw a semantic distinction between anaphoric dependencies and variable binding. As pointed out in the introduction, this distinction deserves to be studies across various constructions, but it is particularly relevant to control, since there is an emerging view that control is not a unitary phenomenon (Cinque 2006; van Urk 2010; Grano 2012; Sheehan to appear; Landau 2014). In derivational terms, it seems that the movement theory of control and the PRO-based theory can both be right, though of different types of control. This is also a traditional view in LFG, which draws a distinction between functional and obligatory anaphoric control. ${ }^{4}$

However, there is one important issue in the literature on partial control that we have not dealt with, namely exactly which verbs license partial control. The details must be left aside here, but note that the present approach is germane to what Landau (2014) has recently proposed, namely that all and only propositional attitude verbs license partial control. This follows naturally if only propositional attitude verbs require a propositional complement (which therefore has its own subject and time arguments) whereas other control verbs take a property complement. It remains to be seen whether such an idea can be upheld in the light of empirical studies on the distribution of partial control across languages.

4 Landau (2014: 6) wrongly implies that the functional/anaphoric control distinction in LFG equals the distinction between obligatory and non-obligatory control in other frameworks, but the notion of obligatory anaphoric control has been present since Bresnan 1982. 
Partial control

\section{References}

Andrews, Avery. 1982. Long distance agreement in Modern Icelandic. In Pauline Jacobson \& Geoffrey Pullum (eds.), The Nature of Syntactic Representation, 1-33. Dordrecht: D. Reidel. doi:10.1007/978-94-009-7707-5_1.

Asher, Nicholas \& Alex Lascarides. 1998. Bridging. Journal of Semantics 15(1). 83-113. doi:10.1093/jos/15.1.83.

Asudeh, Ash. 2005. Control and semantic resource sensitivity. Journal of Linguistics 41(3). 465-511. doi:10.1017/S0022226705003427.

Beaver, David. 1992. The kinematics of presupposition. In Paul Dekker \& Martin Stokhof (eds.), Proceedings of the Eight Amsterdam Colloquium, 17-36. Amsterdam: ILLC.

Belyaev, Oleg \& Dag Trygve Truslew Haug. 2014. Pronominal coreference in Ossetic correlatives and the syntax-semantics interface. In Miriam Butt \& Tracy Holloway King (eds.), Lexical Functional Grammar Conference (LFG14), Stanford.

Boeckx, Cedric, Norbert Hornstein \& Jairo Nunes. 2010. Control as Movement. Cambridge: Cambridge University Press. doi:10.1017/CBO9780511761997.

Bresnan, Joan. 1982. Control and complementation. In Joan Bresnan (ed.), The mental representation of grammatical relations, 282-390. Cambridge, MA: MIT Press.

Cinque, Guglielmo. 2006. Restructuring and Functional Heads. Oxford: Oxford University Press.

Clark, Herbert H. 1975. Bridging. In R. C. Schank \& B. L. Nash-Webber (eds.), Theoretical issues in natural language processing, New York: Association for Computing Machinery.

Geurts, Bart. 2011. Accessibility and anaphora. In Klaus von Heusinger, Claudia Maienborn \& Paul Portner (eds.), Semantics, vol. 2, 1988-2011. Berlin: Mouton de Gruyter. doi:10.1515/9783110255072.1988.

Grano, Thomas. 2012. Control and restructuring at the syntax-semantics interface. University of Chicago $\mathrm{PhD}$ dissertation.

Haug, Dag. 2013. Partial dynamic semantics for anaphora: Compositionality without syntactic coindexation. Journal of Semantics advance access online. doi:10.1093/jos/fft008.

Heim, Irene \& Angelika Kratzer. 1998. Semantics in Generative Grammar. Malden: Blackwell.

Hornstein, Norbert. 1999. Movement and control. Linguistic Inquiry 30(1). 69-96. doi:10.1162/002438999553968.

Hornstein, Norbert. 2003. On control. In Randall Hendrick (ed.), Minimalist Syntax, 6-81. Oxford: Blackwell. doi:10.1002/9780470758342.ch1. 
Kamp, Hans. 1981. A theory of truth and semantic representation. In J. Groenendijk, T. Janssen \& M. Stokhof (eds.), Formal Methods in the Study of Language, 277-322. Amsterdam: Mathematisch Centrum.

Kamp, Hans \& Uwe Reyle. 1993. From Discourse to Logic. Dordrecht: Kluwer. doi:10.1007/978-94-017-1616-1.

Kamp, Hans, Josef van Genabith \& Uwe Reyle. 2011. Discourse Representation Theory. In D. M. Gabbay \& F. Günthner (eds.), Handbook of Philosophical Logic, vol. 15, 125-394. Dordrecht: Springer. doi:10.1007/978-94-007-04855_3.

Landau, Idan. 2000. Elements of Control: Structure and Meaning in Infinitival Constructions. Dordrecht: Kluwer. doi:10.1007/978-94-011-3943-4.

Landau, Idan. 2004. The scale of finiteness and the calculus of control. Natural Language and Linguistic Theory 22(4). 811-877. doi:10.1007/s11049-0044265-5.

Landau, Idan. 2014. A two-tiered theory of control. Available at http://ling.auf.net/ lingbuzz/001937.

Lewis, David. 1979. Attitudes de dicto and de se. Philosophical Review 88(4). 513-543. doi:10.2307/2184843.

Maier, Emar. 2009. Presupposing acquaintance: a unified semantics for de dicto, de re and de se belief reports. Linguistics and Philosophy 32(5). 429-474. doi:10.1007/s10988-010-9065-2.

Muskens, Reinhard. 1996. Combining Montague semantics and discourse representations. Linguistics and Philosophy 19(2). 143-186. doi:10.1007/BF00635836.

Nouwen, Rick. 2003. Plural pronominal anaphora in context. University of Utrecht $\mathrm{PhD}$ dissertation.

Pearson, Hazel. 2013. The sense of self: topics in the semantics of de se expressions. Harvard University $\mathrm{PhD}$ dissertation.

Pearson, Hazel. to appear. The semantics of partial control. Available at http: //semanticsarchive.net/Archive/jIxNDk3M/.

Schlenker, Philippe. 2003. A plea for monsters. Linguistics and Philosophy 26(1). 29-120. doi:10.1023/a:1022225203544.

Sheehan, Michelle. to appear. Portuguese, Russian and the theory of control. In Hsin-Lun Huang, Ethan Poole \& Amanda Rysling (eds.), Proceedings of the 43rd Annual Meeting of the North East Linguistic Society, 115-126. Amherst: GLSA.

Stephenson, Tamina. 2010. Control in centred worlds. Journal of Semantics 27(4). 409-436. doi:10.1093/jos/ffq011.

van Urk, Coppe. 2010. On obligatory control: A movement and pro approach. Available at http://web.mit.edu/cvanurk/www/onobligatorycontrol.pdf.

White, Aaron Steven \& Thomas Grano. 2013. An experimental investigation of 
Partial control

partial control. In Urtzi Etxeberria, Anamaria Fălăuş, Aritz Irurtzun \& Bryan Leferman (eds.), Proceedings of Sinn und Bedeutung 18, University of the Basque Country.

Dag Trygve Truslew Haug

Department of Philosophy, Classics, History of Arts and Ideas

PO Box 1020 Blindern

$\mathrm{N}-0315$ Oslo

Norway

daghaug@ifikk.uio.no 